\title{
ATLANTIC SALMON PHYSIOLOGICAL AND IMMUNE RESPONSE TO AMOEBIC GILL DISEASE AND INSIGHT INTO THE BIOLOGY OF THE AMOEBA
}

\author{
Ottavia Benedicenti ${ }^{1,2 \S}$, Chris Secombes ${ }^{1}$, Una McCarthy ${ }^{2}$, Catherine Collins ${ }^{2 \S}$ \\ ${ }^{1}$ Scottish Fish Immunology Research Centre, Institute of Biological and Environmental Sciences, \\ University of Aberdeen, Tillydrone Avenue, Aberdeen AB24 2TZ, UK \\ ${ }^{2}$ Marine Scotland Science Marine Laboratory, 375 Victoria Rd, Aberdeen AB11 9DB, UK
}

\begin{abstract}
Amoebic gill disease (AGD) is an emerging disease in North European Atlantic salmon (Salmo salar) aquaculture caused by the amoeba Paramoeba perurans. Non-optimal environmental conditions such as increasing water temperature may affect AGD progression. To understand the role of predisposing environmental conditions on the biology of the parasite and on the host physiological and immune response, different clonal cultures of $P$. perurans and AGD infected Atlantic salmon were exposed, respectively, in vitro and in vivo to two different temperatures, $10^{\circ} \mathrm{C}$ and $15^{\circ} \mathrm{C}$.

Outputs from the in vitro experiment are 1) an improved understanding of the impact of temperature on amoebae growth rate during conditions that potentially influence disease development and 2) characterisation of changes in bacterial communities at different temperatures for different clonal cultures. Negative binomial analysis in $\mathrm{R}$ ( $\mathrm{R}$ software, version 3.0.1) showed significant differences in growth rate among clonal cultures over time. Moreover, for each clonal culture, the fraction of amoebae in suspension in seawater showed a higher increase over time at $10^{\circ} \mathrm{C}$, whereas the fraction of amoebae attached to the malt yeast agar (MYA) showed a higher increase over time at $15^{\circ} \mathrm{C} .16 \mathrm{~S}$ MiSeq analysis was also performed to characterise the changes in bacterial communities present in the cultures at the two different temperatures of the in vitro experiment.

The host physiological and immune response to AGD infection during predisposing seasonal weather conditions (summer period) is still not well understood. Atlantic salmon smolts were exposed in vivo to the same temperatures used for the in vitro experiment, $10^{\circ} \mathrm{C}$ and $15^{\circ} \mathrm{C}$, and to an initial concentration of 5000 cells $1^{-1}$ of a clonal culture of $P$. perurans for 3 weeks. Analyses of cortisol, glucose, and lactate concentration in plasma samples, white blood cell (wbc) differential count in blood smears, and gene expression have been performed at different time points after the challenge.

The present results from the temperature dependent AGD infection and in vitro $P$. perurans growth rate studies hint that increased AGD outbreaks during summer periods may not be solely due to increased thermal stress in fish but also to increased amoebae attachment at $15^{\circ} \mathrm{C}$ which cause an increased gill pathology.
\end{abstract}

\section{KEYWORDS}

Paramoeba perurans, AGD, temperature, cortisol implant, stress

${ }^{\S}$ Corresponding authors.

Tel. +44 (0) 1224876544

E-mail address:

r01ob13@abdn.ac.uk

catherine.collins@gov.scot 